\title{
Software package for uncertainty calculations of temperature measurements with thermal cameras
}

\author{
by K. Chrzanowski*, R. Matyszkiel** \\ * Military University of Technology, Inst. of Optoelectronics, 00-908 Warsaw, Poland, \\ kchrza@wat.waw.pl \\ ** Military Institute of Communication, 05-130 Zegrze Pld., Poland
}

\begin{abstract}
:
Difficulties in determining the measurement uncertainty is an important limitation of thermal cameras for the industrial plants and the co-operating accreditation laboratories that have implemented the quality systems according to the international norms ISO 9001-9004 and EN 45001-45003. A software that enables calculations of uncertainties of temperature measurements with thermal cameras was developed and is presented in this paper. It and can help to remove the above mentioned limitation if only the user of the thermal camera can estimate possible dispersion of such parameters of the measurement process like: the object effective emissivity, the effective background temperature, the effective transmittance of the atmosphere and some parameters of the thermal camera.
\end{abstract}

\section{Introduction}

The well known "Guide to the expression of uncertainty in measurement" published in 1992 in the name of the seven main international metrological organizations recommends uncertainty as measure of measurement accuracy and presents methods of its calculations [1]. Publications [2,3] from the National Institute of Standards and Technology and the European Cooperation for Accreditation precise Guide recommendations.

There is a general trend in industry to accept the quality systems according to the international norms ISO 9001-9004 and in co-operating accreditation laboratories - according to norms the EN 45001-45003. According to these documents the industrial plants and accreditation laboratories are required to use reliable measuring devices and to evaluate an uncertainty of measurements and present it in the certificates.

There are a few examples of calculation of uncertainty of different measurement results in the Guide. More examples can be found in Ref.3,4 and in other EA publications. However, there was not published any method of determination of uncertainty of measurement results with thermal cameras. Difficulties in determining the measurement uncertainty is an important limitation of thermal cameras as a verified technique of temperature measurement for the industrial plants and the co-operating accreditation laboratories that have implemented the quality systems according to the international norms ISO 9001-9004 and EN 4500145003. A mathematical model of uncertainty of temperature measurement with thermal cameras was recently developed $[5,6]$. On the basis of this model a software that enables calculations of uncertainties of temperature measurements with thermal cameras was developed and is presented in this paper. It and can help to remove the above mentioned limitation if only the user of the thermal camera can estimate possible dispersion of such parameters of the measurement process like: effective object emissivity, effective background temperature, effective transmittance of the atmosphere and some parameters of the thermal camera.

\section{Model of uncertainty of thermal cameras}

The combined standard uncertainty of temperature measurement with thermal cameras can be calculated using the square root of the sum of squares partial uncertainties due to possible random variations of the real object effective emissivity, the real effective tempera- 
ture of the background, the real effective transmittance of the atmosphere, and the unknown errors due to sources within the thermal camera using the following formula $[5,6]$

$$
\begin{aligned}
& u_{c}\left(T_{o b}\right)=\sqrt{u_{\varepsilon}^{2}+u_{T}^{2}+u_{\tau}^{2}+u_{i n}^{2}} \\
& =\sqrt{\left(c_{\varepsilon} u\left(\varepsilon_{r}\right)\right)^{2}+\left(c_{T} u\left(T_{b a(r)}\right)\right)^{2}+\left(c_{\tau} u\left(\tau_{a(r)}\right)\right)^{2}+u\left(\Delta T_{i n}\right)^{2}}
\end{aligned}
$$

where $T_{o b}$ is the output quantity, $u\left(\varepsilon_{r}\right)$ is the standard uncertainty of determination of the object real effective emissivity $\varepsilon, u\left(T_{b a(r)}\right)$ is the standard uncertainty of determination of the real effective background temperature $T_{b a(r)}, u\left(\tau_{a(r)}\right)$ is the standard uncertainty of determination of the real effective transmittance of the atmosphere $\tau_{a(r)}, u\left(\Delta T_{\text {in }}\right)$ is the standard uncertainty due to intrinsic errors of the thermal camera.

The coefficients $C_{\varepsilon}, C_{T}, C_{\tau}$ can be calculated as

$$
\begin{gathered}
c_{\varepsilon}=-\frac{\left.\int_{d} \frac{s y s(\lambda)}{\lambda^{5}\left[\exp \left(c_{2} / \lambda T_{\text {out }}\right)-1\right]} d \lambda-\int_{\lambda_{d}} \frac{\operatorname{sys}(\lambda)}{\lambda^{5}\left[\exp \left(c_{2} / \lambda T_{\text {ba(a) }}\right)-1\right]} d \lambda\right)}{\int_{\lambda d} \frac{\varepsilon_{a} \operatorname{sys}(\lambda) \exp \left(c_{2} / \lambda T_{\text {out }}\right)}{\lambda^{6} T_{\text {out }}^{2}\left[\exp \left(c_{2} / \lambda T_{\text {out }}\right)-1\right]^{2}} d \lambda}, \\
\left.\int_{T} \frac{c_{2} \exp \left(c_{2} / \lambda T_{\text {ba }(a)}\right)\left(1-\varepsilon_{a}\right) \operatorname{sys}(\lambda)}{\lambda_{d}^{6} T_{\text {ba(a) }}^{2}\left[\exp \left(c_{2} / \lambda T_{\text {ba }(a)}\right)-1\right]} d \lambda\right) \\
\left.\int_{\tau}=-\frac{\int_{d} \frac{\varepsilon_{a} s y s(\lambda) \exp \left(c_{2} / \lambda T_{\text {out }}\right)}{\lambda^{6} T_{\text {out }}^{2}\left[\exp \left(c_{2} / \lambda T_{\text {out }}\right)-1\right]^{2}} d \lambda}{\lambda^{5}\left[\exp \left(c_{2} / \lambda T_{\text {out }}\right)-1\right]} d \lambda-\int_{\lambda_{d}} \frac{\left(1-\varepsilon_{a}\right) \operatorname{sys}(\lambda)}{\lambda^{5}\left[\exp \left(c_{2} / \lambda T_{\text {ba }}(a)-1\right]\right.} d \lambda\right) \\
\int_{\lambda d} \frac{\varepsilon_{a} \tau_{a(a)} \operatorname{sys}(\lambda) \exp \left(c_{2} / \lambda T_{\text {out }}\right)}{\lambda^{6} T_{\text {out }}^{2}\left[\exp \left(c_{2} / \lambda T_{\text {out }}\right)-1\right]^{2}} d \lambda
\end{gathered}
$$

where the $c_{2}$ is the Planck constant, $s y s(\lambda)$ is the spectral sensitivity function of the thermal camera, $\varepsilon_{a}$ is the assumed by the user effective emissivity of the tested object, $T_{b a(a)}$ is the assumed effective background temperature, $\tau_{a(a)}$ is the assumed effective transmittance of the atmosphere.

We can see in Eqs. (1-2) that in order to calculate the combined standard uncertainty $u_{c}\left(T_{o b}\right)$ it is necessary to know: the output temperature $T_{\text {out, }}$ the spectral sensitivity function of the thermal camera $\operatorname{sys}(\lambda)$, the assumed value of the effective emissivity $\varepsilon_{a}$ and its standard uncertainty $u\left(\varepsilon_{r}\right)$, the assumed value of the effective background temperature $T_{b a(a)}$ and the standard uncertainty $u\left(T_{b a(r)}\right)$, the assumed value of the effective transmittance of the atmosphere $\tau_{a(a)}$ and the standard uncertainty $u\left(\tau_{a(r)}\right)$, and the intrinsic uncertainty $u\left(\Delta T_{\text {in }}\right)$ of the thermal camera.

Let us now find whether is it possible to determine these parameters.

The output temperature $T_{\text {out }}$ is the reading temperature of the camera and is always known to the camera user. The sys $(\lambda)$ can be found in camera manual or other literature. $\varepsilon_{a(a)}, T_{b a(a)}$, and the $\tau_{a(a)}$ are values assumed by the user during the measurement process.

Users of thermal cameras usually do not know the uncertainties $u\left(\varepsilon_{r}\right), u\left(T_{b a(r)}\right), u\left(\tau_{a(r)}\right)$. However, they can usually be estimated as the users can almost always estimate bounds of 
the random variables $\varepsilon_{r}, T_{b a(r)}, \tau_{a(r)}$. This means that it is known that the real effective emissivity $\varepsilon_{r}$ is located within the range $\left[\varepsilon_{a}-\Delta \varepsilon, \varepsilon_{a}+\Delta \varepsilon\right]$, the real effective background temperature $T_{b a(r)}$, is located within the range $\left[T_{b a(a)}-\Delta T_{b a}, T_{b a(a)}+\Delta T_{b a}\right.$, the real effective transmittance $\tau_{a(r)}$ is located within the range $\left[\tau_{a(a)}-\Delta \tau, \tau_{a(a)}-\Delta \tau\right]$.

Although the users can usually estimate bounds of the random variables $\varepsilon_{r}, T_{b a(r)}, \tau_{a(r)}$ they rarely can estimate type of probability distribution of these quantities. Therefore, let us assume a uniform distribution of these quantities within the earlier specified ranges as such an assumption is commonly made when there is no specific knowledge about possible values of quantity $x$ within a certain range [1]. Then the uncertainties $u\left(\varepsilon_{r}\right), u\left(T_{b a(r)}\right), u\left(\tau_{a(r)}\right)$ can be calculated as

$$
u\left(\varepsilon_{r}\right)=\frac{\Delta \varepsilon}{\sqrt{3}} \quad u\left(T_{b a(r)}\right)=\frac{\Delta T_{b a}}{\sqrt{3}} \quad u\left(\tau_{a(r)}\right)=\frac{\Delta \tau}{\sqrt{3}} .
$$

Nowadays, manufactures of thermal cameras present very rarely the intrinsic standard uncertainty $u\left(\Delta T_{\text {in }}\right)$ of the thermal camera. However, we can expect that this parameter will be commonly presented in catalogs of thermal cameras in near future.

When the intrinsic standard uncertainty $u\left(\Delta T_{\text {in }}\right)$ is not supplied by the manufacturer then it can be determined on the basis of a set of parameters measured by the user: the minimum error ME, the noise generated error NGE, the digital temperature resolution DTR, the temperature stability TS, the measurement uniformity MU, and the measurement spatial resolution MSR.

The minimum error ME is defined as a range around the output temperature $T_{\text {out }}$ in which the true temperature $T_{o b}$ is located when the measurements are carried out in conditions identical with the conditions during calibration of the thermal camera. The noise generated error NGE is defined as the standard deviation of the output temperature dispersion due to camera noise. The digital temperature resolution DTR is defined as the smallest difference between two temperature levels that can be distinguished because of the limited resolution of the digital channel of the thermal camera.

The temperature stability $T S$ is defined as a range in which the results of the measurements carried out in different environment temperatures, within limits determined by the camera manufacturer, are located. The measurement uniformity $M U$ is defined as a range in which the results of the measurements are located when the tested object is located at different places within the field of view of the camera. The measurement spatial resolution MSR is defined as the minimum angular dimension of the tested object when there is still no the influence of limited size of this object on temperature measurement results.

Description of the calculation method of the $u\left(\Delta T_{i n}\right)$ on the basis of the measured ME, NGE, DTR, TS, MU, MSR is presented in Ref. 12.

\section{Description of the Thermax software}

The developed software package Thermax requires from its user to make 2 following steps to calculate the combined standard uncertainty $u_{c}\left(T_{\text {out }}\right)$ of the output temperature $T_{\text {out }}$.

1. Insert parameters characterising the measurement conditions:

- output temperature $T_{\text {out }}$,

- the temperature span of the thermal camera used during measurements,

- angular size of the tested object,

- $\quad$ assumed by the user value of the object emissivity $\varepsilon_{a}$

- assumed by the user value of the background temperature $T_{b a(a)}$,

- $\quad$ assumed by the user value of the effective transmittance of the atmosphere $\tau_{a(a)}$,

- some indications of possible dispersions of the parameters $\varepsilon_{a,}, T_{b a(a),} \tau_{a(a)}$ (the user can insert bounds within which these parameters are expected to lie and type of distribution, or to insert the standard deviation).

2. Insert parameters characterising the thermal camera 
- the spectral sensitivity function sys $(\lambda)$,

- the intrinsic uncertainty.

The user of this software can choose between typical spectral sensitivity function $\operatorname{sys}(\lambda)$ for 3-5 $\mu \mathrm{m}$ or 8-12 $\mu \mathrm{m}$ thermal cameras or define its own spectral sensitivity function sys $(\lambda)$. In case of the intrinsic uncertainty of the thermal camera he has two options. First, to insert directly the intrinsic uncertainty $u\left(\Delta T_{\text {in }}\right)$. The form $\mathrm{x} \%$ of the $T_{\text {out }}$ but not less that $\mathrm{y}^{\circ} \mathrm{C}$ is the default function for presentation of the intrinsic uncertainty $u\left(\Delta T_{i n}\right)$. Second, the user can insert the presented earlier parameters: the minimum error $M E$, the noise generated error $N G E$, the digital temperature resolution DTR, the temperature stability $T S$, and the measurement uniformity $M U$, the measurement spatial resolution MSR and then the intrinsic uncertainty $u\left(\Delta T_{\text {in }}\right)$ will be calculated by the software.

On the basis of the inserted data Thermax calculates standard uncertainty or the extended uncertainty of determination of the output temperature. As we can see above there are quite many parameters of the thermal camera required for the calculations of the uncertainty of measurements with these cameras. However, all these parameters can be determined by proper testing of the camera by the user or, at worst case, they can be roughly estimated by the user.

The software Thermax works in the following systems: Windows NT, Windows 98, Windows 95. It enables calculations of the combined standard uncertainty and the expanded uncertainty. Two version of reports produced by Thermax : the simplified version (the estimate of the output quantity and its standard uncertainty of its extended uncertainty) or the full version (the table recommended by EA). The report may be printed, transferred via clipboard or to data files. Two different formats are supported (Text only format or HTML). The latter format is particularly important as it enables possibility to create sophisticated reports in a well known format and to run such applications like Internet Explorer, Netscape Navigator to interpret it and to print from this applications.

\section{Calculations}

Let us carry out calculations for the case of the measurement conditions and the camera parameters presented in Tab.1. It seems that similar situations like presented in Tab.1 can be met in many applications of thermal cameras.

The results of the calculations of the combined standard uncertainty of the output temperature using the Thermax software are shown in Tab.2.

The calculations of combined standard uncertainty of the output temperature using the Thermax software for the situation presented in Tab.1 or any other can be carried out within a second. Therefore, the software Thermax can save user of thermal cameras many hours of time spent on estimation of uncertainty of measurements with these cameras.

It is difficult to determine accuracy of the calculations carried out using the Thermax software. We can say that the software is based on a mathematical model that was developed in according to the recommendations of the main international metrological organisations presented in Ref.1. However, accuracy of the Thermax software depends not only on the mathematical model but also on the input data inserted by the user of this software who is expected to estimate the possible dispersion of the assumed values of the object effective emissivity, the effective background temperature, the effective atmospheric transmittance, and to determine intrinsic uncertainty of the thermal camera. The accuracy of determination of these parameters by the user can vary significantly depending on the user and applications. However, it seems that for a case of an experienced user of thermal cameras and in typical application it is possible to determine the combined standard uncertainty of the output temperature with errors smaller than $20-50 \%$. Such a level of possible error of determination of the uncertainty is typically acceptable in many of the industrial plants and laboratories that implemented the quality systems according the international standards ISO 9000 and EN 45000. 


\section{Conclusions}

The developed software can be a useful tool for the users of thermal cameras enabling them estimation of uncertainty of temperature measurements, what is particularly important in the industrial plants and laboratories that implemented the quality systems according the international standards ISO 9000 and EN 45000.

\section{References}

[1] Guide to the expression of uncertainty in measurement, International Organisation for Standardisation-International Electrotechnical Commission-International Organisation of Legal Metrology-International Bureau of Weights and Measures, TAG 4/WG 3 (1993).

[3] Expression of the uncertainty of measurement in calibration, EAL-R2 (19997).

[4] Expression of uncertainty of measurement in calibration, Supplement to EAL-R2

[5] K. Chrzanowski, J. Fischer, M. Matyszkiel, Testing and evaluation of thermal cameras for absolute temperature measurement, Optical Engineering, 39,9 (2000)

[6] K. Chrzanowski, J. Fischer, M. Matyszkiel, Evaluation of uncertainty of temperature measurement with thermal cameras, Optical Engineering (accepted for publication).

Tab.1. The measurement conditions and the camera parameters used in the calculations

\begin{tabular}{|l|l|}
\hline \multicolumn{2}{|c|}{ Measurement conditions } \\
\hline output temperature $T_{\text {out }}$ & $400^{\circ} \mathrm{C}$ \\
\hline camera temperature span during measurements & $100^{\circ} \mathrm{C}$ \\
\hline angular size of the tested object & $150 \mathrm{mrad}$ \\
\hline $\begin{array}{l}\text { assumed value of the object effective emissivity } \varepsilon_{a} \text { and } \\
\text { its possible dispersion }\end{array}$ & $0.7 \pm 0.1$ (uniform distribution) \\
\hline $\begin{array}{l}\text { assumed value of the background temperature } T_{b a(a)} \text { and } \\
\text { its possible dispersion }\end{array}$ & $40^{\circ} \mathrm{C} \pm 10^{\circ} \mathrm{C}$ \\
\hline $\begin{array}{l}\text { assumed value of the effective transmittance of the at- } \\
\text { mosphere } \tau_{\text {a(a) }} \text { and its possible dispersion }\end{array}$ & $0.95 \pm 0.3$ \\
\hline \multicolumn{2}{|c|}{ Camera parameters } \\
\hline spectral sensitivity sys $(\lambda) \quad \begin{array}{l}\text { typical for } 8-12 \mu \mathrm{m} \text { thermal cam- } \\
\text { eras }\end{array}$ \\
\hline intrinsic uncertainty of the camera & $\begin{array}{l}2 \% \text { of the output temperature but } \\
\text { not less than } 2^{\circ} \mathrm{C}\end{array}$ \\
\hline
\end{tabular}

Tab.2. Results of the calculations

\begin{tabular}{|l|l|}
\hline Source of uncertainty & Partial standard uncertainty \\
\hline errors of determination of the object effective emissivity & $35.3^{\circ} \mathrm{C}$ \\
\hline $\begin{array}{l}\text { errors of determination of the background effective tem- } \\
\text { perature }\end{array}$ & $0.68^{\circ} \mathrm{C}$ \\
\hline $\begin{array}{l}\text { errors of determination of the effective transmittance of the } \\
\text { atmosphere }\end{array}$ & $8.1^{\circ} \mathrm{C}$ \\
\hline intrinsic uncertainty of the thermal camera & $8^{\circ} \mathrm{C}$ \\
\hline Output temperature & $\begin{array}{l}\text { Combined standard uncer- } \\
\text { tainty }\end{array}$ \\
\hline $400^{\circ} \mathrm{C}$ & $37.1^{\circ} \mathrm{C}$ \\
\hline
\end{tabular}



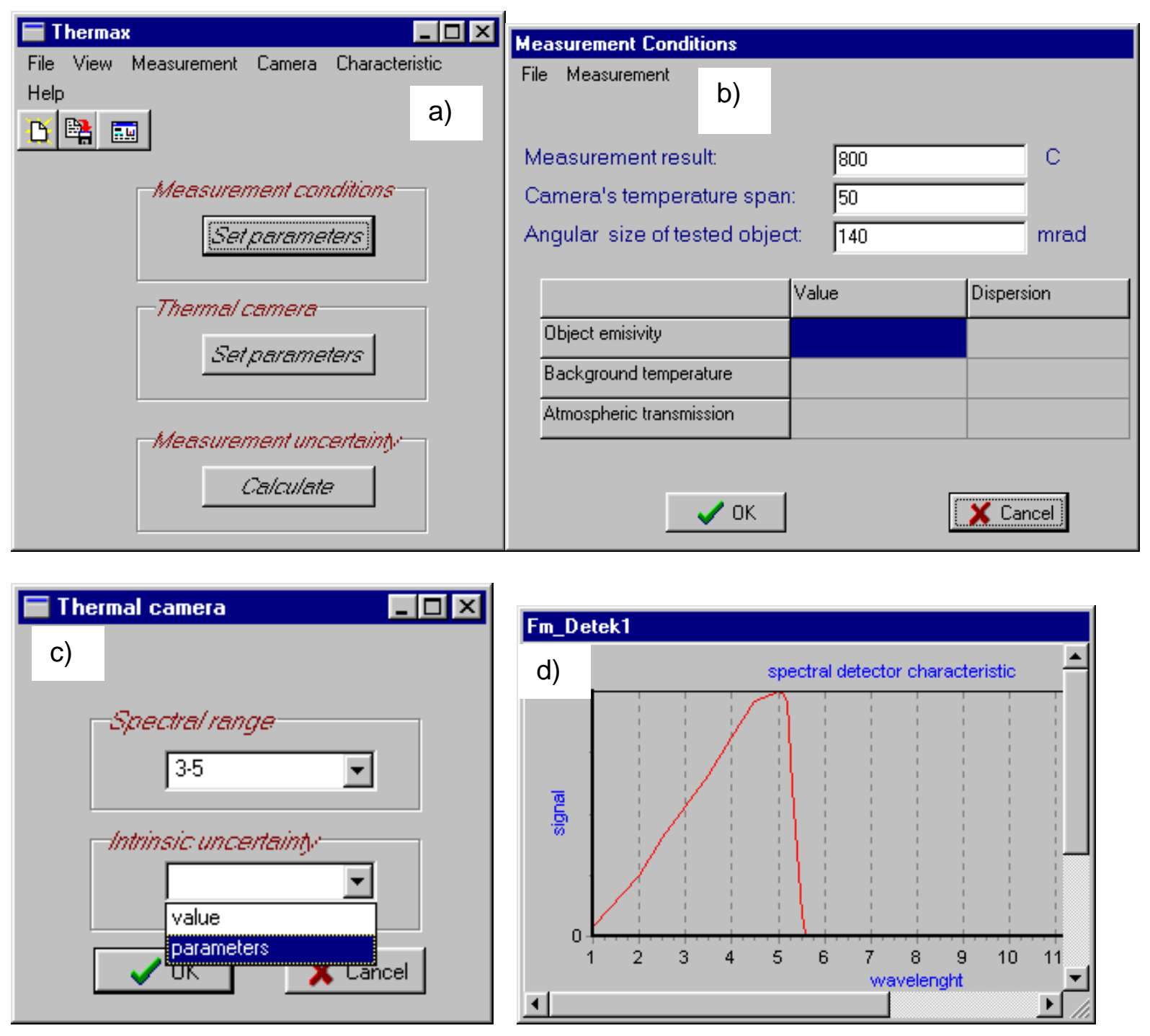

\section{Measurement uncertainty}

e)

\begin{tabular}{|c|c|c|c|}
\hline & Sensitivity coefficient & \multicolumn{2}{|c|}{ Uncertainty component } \\
\hline Emisivity & 604.0974 & \multicolumn{2}{|c|}{34.8806} \\
\hline Background temperature & 0,02894 & \multicolumn{2}{|c|}{0,1671} \\
\hline Atmospheric transmission & 335.6096 & \multicolumn{2}{|c|}{5.8128} \\
\hline Intrinsic uncertainty & 1 & \multicolumn{2}{|l|}{16} \\
\hline \multicolumn{3}{|c|}{ Measurementresulis. } & 800 \\
\hline \multicolumn{3}{|c|}{ Combined uncentaintl atmeasurement is: } & $38.8133 \mathrm{C}$ \\
\hline
\end{tabular}

Fig.1. View of some forms used in software Thermax : a) the introductory form, b) the basic measurement conditions form, $c$ ) the basic camera parameters form, $d$ ) the camera spectral sensitivity form, e) the final output form. 